\title{
Assessment of environmental effects of deep ocean mining of manganese nodules
}

\author{
R. E. Burns \\ DOMES Project/NOAA, 7600 Sand Point Way N. E.; \\ Seattle, Washington 98115, USA
}

\begin{abstract}
Deep ocean mining for manganese nodules is under development by several multinational mining consortia. Initial estimates of probable environmental effects of this mining were made before any actual operations were conducted. Recent pilot mining tests near Hawaii have provided an opportunity to verify and modify the initial estimates. The two principal initial impacts of mining are in the near surface water associated with increased turbidity caused by mining

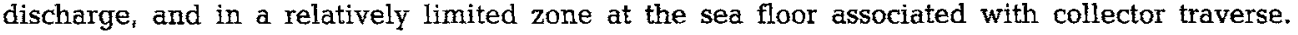
Examples are presented of estimates of reduction in primary production in the turbid plume as well as the contrasting effect of increased nutrients. Effects of the mining collector on benthic populations are considered. The limitations of extrapolation of results obtained during pilot mining tests are discussed.
\end{abstract}

\section{INTRODUCTION}

The basic goal of the Deep Ocean Mining Effects Study (DOMES) is to assess the probable environmental consequences of commercial-scale mining of manganese nodules. Beginning in 1975, prior to commencement of any mining activity, the DOMES pre-mining field efforts were concentrated at three sites (Fig. 1), which had been selected as typical mining sites representative of the environmental regimes that are present in the region.

The area of interest is approximately $5 \mathrm{~km}$ deep, and the environmental character of both water column and sediment/nodules has been summarized by Bischoff \& Piper (1979). The basic pre-mining work of the DOMES Project has been summarized by Ozturgut et al. (1978). The results reported here represent part of an increasing worldwide effort to examine probable environmental effects by using pre-mining estimates, in combination with monitoring of those pilot-mining tests which take place prior to onset of commercial-scale mining. The only undertaking in deep water similar to the DOMES work is associated with development of the mining of metalliferous muds from the Red Sea (Mustaffa \& Amann, 1978).

\section{MINING TECHNIQUE}

Although there are several mining consortia, most of them are planning to use hydraulic lift systems to recover the manganese nodules (Fig. 2). Generically, these 


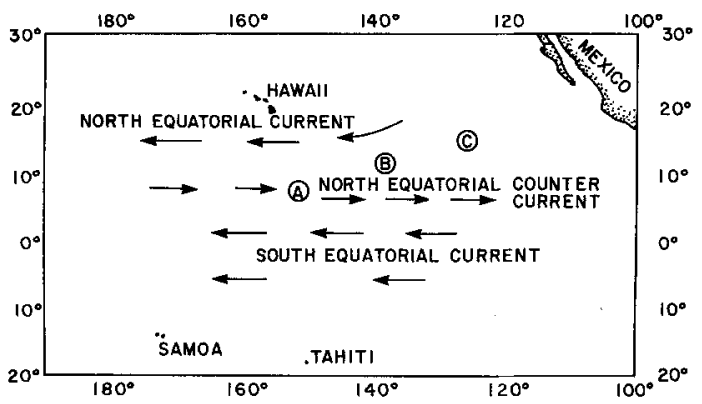

Fig. 1. General circulation scheme in the Deep Ocean Mining Effects Study (DOMES) region $(\mathrm{A}, \mathrm{B}, \mathrm{C})$

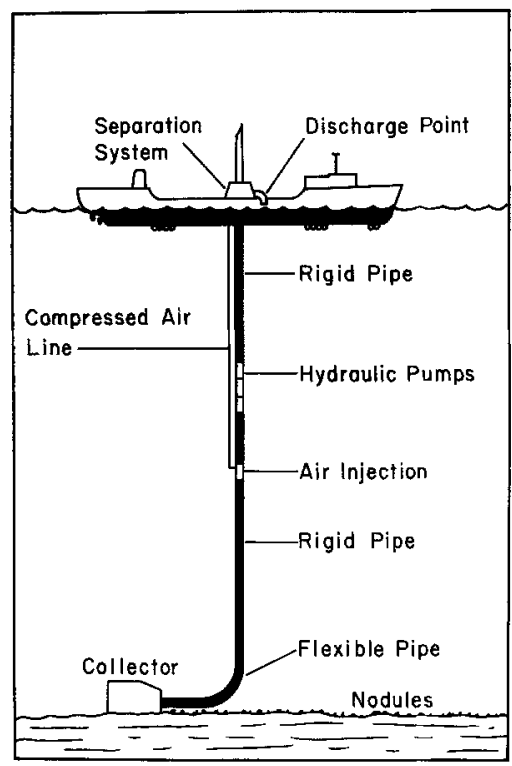

Fig. 2. Schematic diagram of the mining system used during November 1978 tests by Deepsea Ventures, Inc.

hydraulic lift systems use a collector which moves along the sea floor and scrapes nodules and sediment. from the upper few centimeters. Within the collector, nodules and most of the sediment are separated, leaving the sediment behind as the nodules are raised by the lift system. At the surface, further separation of the nodules occurs, and the unwanted water and sediment are discharged over the side of the mining vessel into the surface water.

This type of mining system results in three basic impacts on the marine environment: (1) at the sea floor, the collector removes both nodules and sediment in an area along its track - this is the collector contact zone; (2) the initial separation of nodules and sediment at the collector results in rejection of most of the sediment in a cloud of 
resuspended material - this resuspended material forms the benthic plume; and (3) at the sea surface, the unwanted material which forms the mining ship's overside discharge is the source of the surface plume.

\section{PRE-MINING ASSESSMENT OF PROBABLE IMPACT}

Analysis of the engineering characteristics of the mining system resulted in initial estimates of throughputs for mining operations (Table 1). These estimates were reviewed with representatives of the mining consortia, and a "standard" system was defined based on a daily production of 5000 metric tons of nodules.

Table 1. Standard mining system throughputs per day for 5000-ton/day production unit

\begin{tabular}{|llll|}
\hline Components & Input & Benthic discharge & Surface discharge $^{++}$ \\
\hline Nodules & $5200 \mathrm{mt}$ (dry) & $150 \mathrm{mt}$ (dry) & $50 \mathrm{mt}$ (dry) \\
& $3715 \mathrm{~m}^{3}$ & $105 \mathrm{~m}^{3}$ & $35 \mathrm{~m}^{3}$ \\
Sediment & $3.6 \times 10^{4} \mathrm{mt}$ & $3.5 \times 10^{4} \mathrm{mt}$ & $1000 \mathrm{mt}$ \\
& $1.8 \times 10^{4} \mathrm{~m}^{3}$ & $1.75 \times 10^{4} \mathrm{~m}^{3}$ & $500 \mathrm{mt}^{3}$ \\
Biota $\left(.87 \mathrm{~g}^{-2}\right)$ & $783 \mathrm{~kg}$ & $760 \mathrm{~kg}$ & $23 \mathrm{~kg}$ \\
Bottom water & $5.8 \times 10^{4} \mathrm{~m}^{3}$ & - & - \\
Interstitial water & $4.2 \times 10^{4} \mathrm{~m}^{3}$ & - & - \\
Total water & $1 \times 10^{5} \mathrm{~m}^{3}$ & $8.10^{4} \mathrm{~m}^{3}$ & $2 \times 10^{4} \mathrm{~m}^{3}$ \\
* Basic components of the total mining system throughputs & \\
** Components entering the collector at sea floor & \\
+ Components discharged within 20 m of sea floor \\
++ Components discharged at sea surface \\
\hline
\end{tabular}

The throughputs of this standard system, the expected dispersion of the discharge, and the properties and behavior of the water column in the region permitted initial estimates of the probable effect of mining. These included both "direct effects" (changes in physical/chemical composition attributable to mining) and "indirect effects" (effects on the marine biota of the changed physical/chemical composition).

Time/length scales for direct effects were estimated, which consider both shortterm/near-field effects (times of the order of hours to days and distances of the order of kilometers to tens of kilometers from the mining ship) and long-term/far-field effects (time of the order of years to decades and distances of the order of ocean basin dimensions). These two scales appear to be appropriate in considering both the effects of the particular mining ship's discharge plumes and the possible long-term cumulative effects of a full-scale mining operation.

Using the standard system throughputs as input terms, the initial mixing and subsequent dispersion of the surface discharged material were examined, and the analysis predicted negligible changes above ambient. Furthermore, it was estimated that there would be minor localized reduction in primary production resulting from the light reduction by the suspended particulates, but there would be negligible enhancement of primary production due to addition of nutrients. 
At the sea floor, we expected relatively heavy damage to the benthic biota in the collector contact zone, additional impact in an area of heavy resedimentation associated with the collector contact zone, and the existence of a detectable benthic plume for distances of tens of kilometers away from the collector.

The pre-mining estimates of probable environmental effects presented by Ozturgut et al. (1978) provided a basis for designing a monitoring program which was applied during the initial pilot-mining tests.

\section{MONITORING OF THE PILOT-MINING TESTS}

An opportunity to verify the pre-mining estimates of probable environmental effects was provided by the pilot-mining tests of three of the mining consortia. These took place between early 1978 and early 1979 and involved considerable time at sea. Unfortunately, because of the developmental nature of the mining tests, only five full days of real mining took place, but all of these were monitored.

One part of the monitoring program involved collection of mining ship discharge in order to evaluate the accuracy of our pre-mining estimates of composition and rate of the discharge. Although the pilot-mining tests were approximately $1 / 5$ the planned commercial scale in volume of discharge, the earlier estimates of the composition of the discharge proved to be accurate, with the exception that the content of abraded nodules was somewhat higher than the original estimate.

The waste material is discharged through a pipe over the side of the mining ship. Initial mixing, due to both convective settling and turbulence associated with ship movement, resulted in a rapid initial reduction in the particulate concentration over distances of only a few tens of meters away from the discharge point. After this relatively rapid initial activity, there was still a detectable surface plume which spread downstream from the mining ship.

In order to evaluate our initial estimates of the dispersion of material in the surface plume, we implanted drogues which were set to move with the upper $30 \mathrm{~m}$ of the surface mixed layer. Once these drogues were set, they became a reference point which enabled sampling of the plume at increasing age and distance from the mining ship.

\section{RESULTS FROM MONITORING THE SURFACE PLUME}

The primary experimental design for examining the surface plume involved collection of samples which permitted comparison of concentrations inside the plume with ambient concentrations outside the plume. The samples were collected by continuous sampling at 3-, 5-, 8-, and 13-m depths during traverses across the plume at increasing plume age and by vertical casts through the plume to a depth below the pycnocline.

As suggested by the pre-mining estimates, in-plume differences in dissolved elements were indistinguishable from ambient values in samples obtained more than a few meters from the discharge point. On the other hand, the resuspended particulates in the surface plume were markedly above ambient concentrations and provided the most useful set of data obtained during the monitoring. 


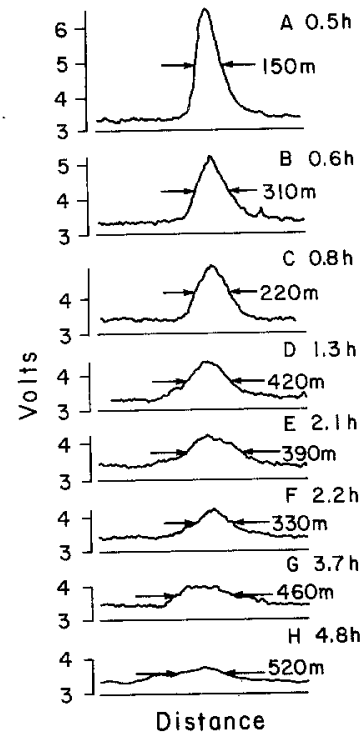

Fig. 3. A sequence of nephelometer traces through the OMI plume at increasing plume ages. Transit speed variations ( 2.3 to 4.7 knots) lead to variations in the scale on the abscissa

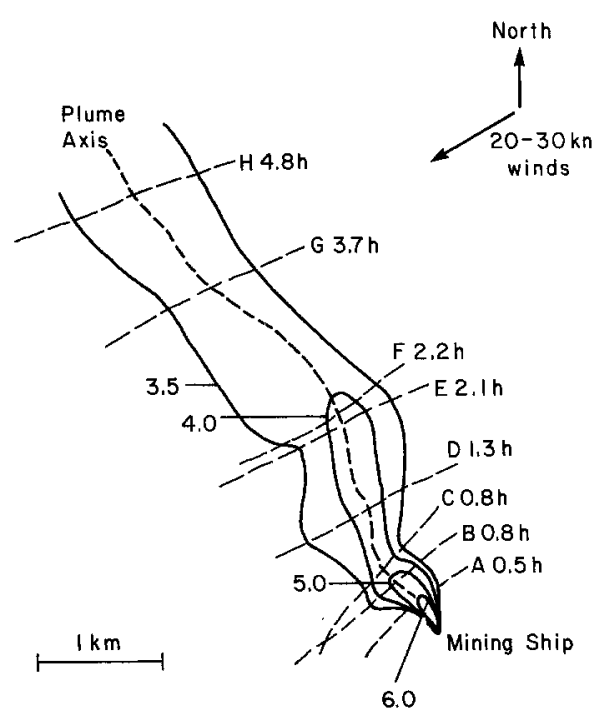

Fig. 4. Planar reconstruction of the steady state OMI mining plume. Contour levels are in volts

A series of traverses (Fig. 3) across the plume was made using a nephelometer at increasing plume age (increasing age from $\mathrm{A}-\mathrm{H}$ ). The decrease in peak amplitude indicates a decrease in the particulate concentration with increasing age. The "spread" of the base of the curve indicates a spread of the particulate plume with increasing age.

Based on the data from the nephelometer traverses, a reconstruction of the surface plume has been made. This indicates that a particulate plume was detectable by this technique for a period of several hours after discharge and at distances of the order of ten kilometers from the mining ship (Fig. 4).

Other techniques, primarily chemical analyses of the particulates, permit identification of the surface plume for about a day to a day-and-a-half. The chemical analyses of particulates from the "plume" have higher $\mathrm{Fe}, \mathrm{Al}$, and $\mathrm{Mn}$ content and lower $\mathrm{Ca}$ than ambient water.

From the data collected during the plume sampling, some additional information has been derived. Figure 5 shows the measured relative changes in the discharged suspended particulate concentrations in the upper $6 \mathrm{~m}$ of the plume at increasing plume age. This indicates as much as $60-70 \%$ decrease in particulate concentration at $6-\mathrm{m}$ depth in the first five hours after discharge. These data, plus additional analyses of the settling velocity of the discharged material, indicate that the settling velocities, based on standard size analyses of discharged material, are much less than those actually measured and observed during the pilot-mining tests. This has a direct implication on the residence times of the discharged material and on estimates of their possible long-term effects in the surface mixed layer.

Examination of the vertical distribution of particulates was made with vertical casts 


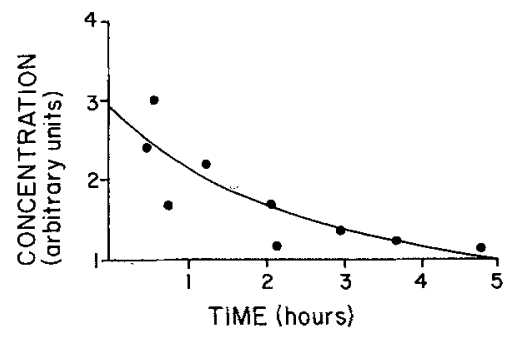

Fig. 5. Measured relative changes of discharged suspended particulate matter concentration in the top $6 \mathrm{~m}$ of the water column of DOMES Site A, May 4, 1978

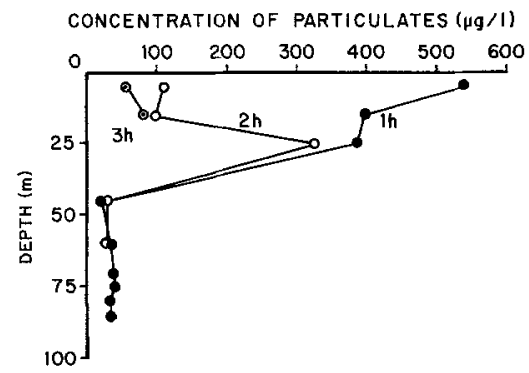

Fig. 6. Particulate concentrations in 1-, 2-, and 3-h-old plume water during Deepsea Ventures, Inc., test on November 1978

in the plume as the plume aged. Figure 6 indicates total particulate concentrations at plume ages of 1,2 , and $3 \mathrm{~h}$. Note the decreasing maximum concentration with increasing age. The maximum values are not at the surface and act as evidence of settling of the plume. However, both the 1- and 2-h data indicate that the settling has not been more than 30 to $40 \mathrm{~m}$, since the deeper values are comparable with ambient values measured prior to mining.

Monitoring the surface plumes generated by the pilot-mining tests was difficult because of the limited time period that each plume was actually generated. In addition, even considering the less-than-full-scale nature of the tests, the plumes were much smaller than anticipated. The settling velocity spectrum of the discharged particulates was higher than pre-mining estimates and, consequently, the duration of the plume (both temporal and spatial) had been initially overestimated. On the other hand, earlier conclusions about the dissolved materials in the discharge appear correct, since a series of experiments contrasting in-plume and ambient conditions all indicated no detectable differences.

\section{EFFECTS OF THE SURFACE PLUME ON BIOTA}

In addition to examining direct physical/chemical effects of surface discharge, several experiments were conducted to evaluate the indirect effect - that is, the effect of these physical/chemical changes on the biota.

Figure 7 indicates the measured light intensity within and below a 1-h-old plume. The open circles are values from within and below the plume, and the black circles are values from ambient water outside the plume. As expected, the increased particulate concentration in the plume causes a marked reduction in the amount of light penetrating into the surface mixed layer. Note also that the plume effect on increasing light attenuation is present to depths of only about $25 \mathrm{~m}$, and below this depth, the light attenuation with depth is comparable to the observed ambient conditions.

An estimate (Fig. 8) has been made of the reduction in primary production caused by the observed reduction in light available in the surface mixed layer. Ambient primary production was measured by a series of in situ experiments, and the reduced values are based on ambient production at light levels comparable to the light levels observed under the plume. This indicates an in-plume reduction of about $45 \%$ from ambient values. 


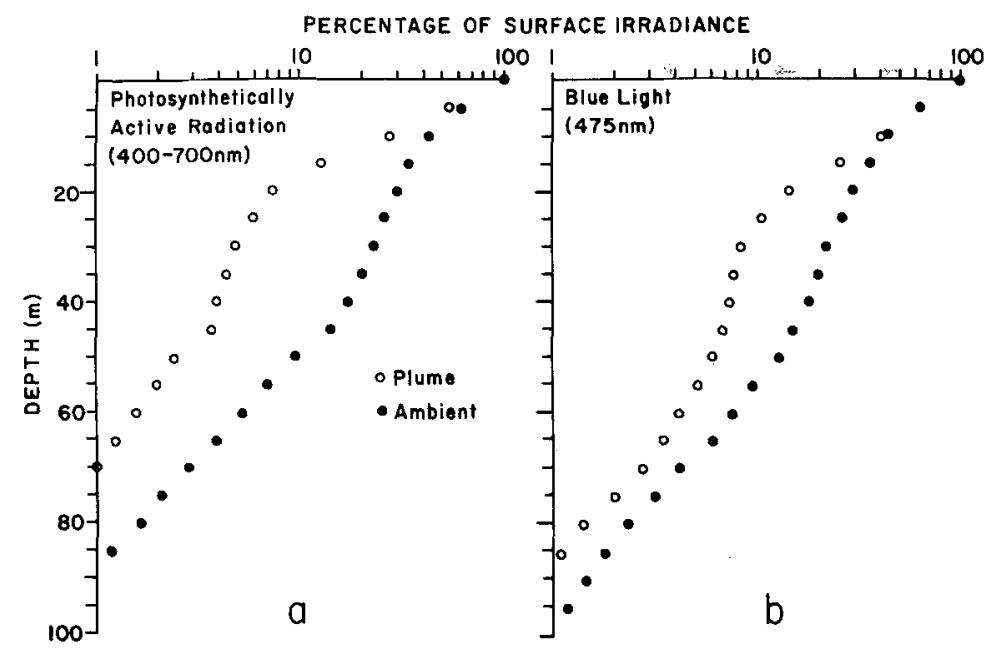

Fig. 7. Profiles of (a) photosynthetically active radiation and (b) blue light in ambient and 1-h-old plume water
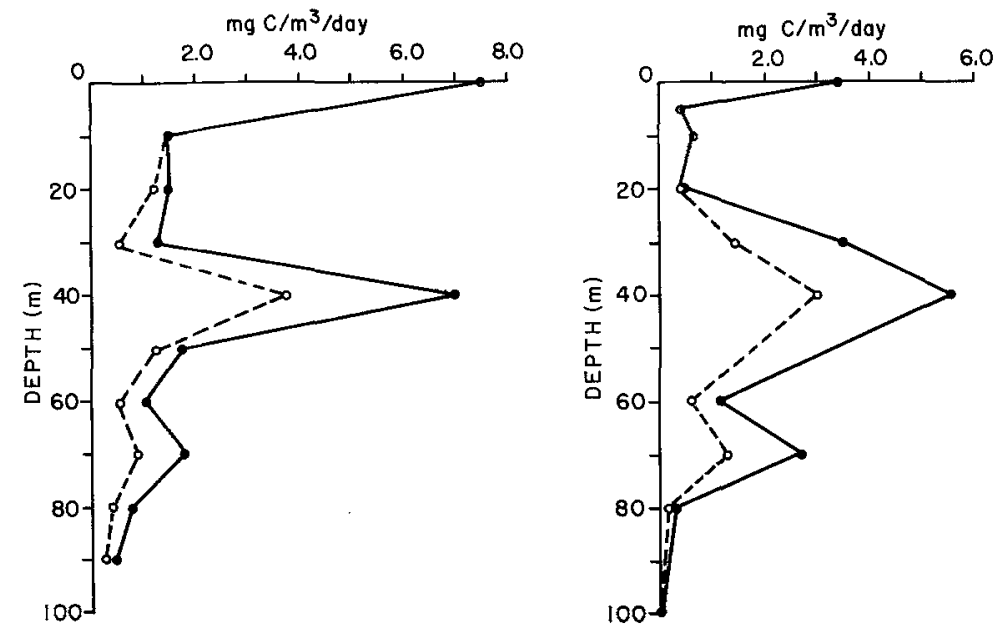

Fig. 8. Measured production rates in the ambient water (solid circles) and estimated production rates at the same station as a result of reducing photosynthetically active radiation (PAR) by the amounts corresponding to the reduction of PAR in 1-h-old plume water (open circles)

This is a recognizably crude estimate, but in situ production experiments could not be conducted within the plume because of its small size and short temporal duration. On the other hand, the estimate appears to be a reasonable approximation of an upper limit since it is based on 1-h-old plume. With additional plume aging, both the particulate concentration and the resultant attenuation of light will diminish rapidly.

In addition to the inhibiting effect of decreased available light, the stimulating effect of added nutrient was examined in a series of ondeck incubation experiments. The effect of varying concentrations of discharge was examined under comparable light conditions 


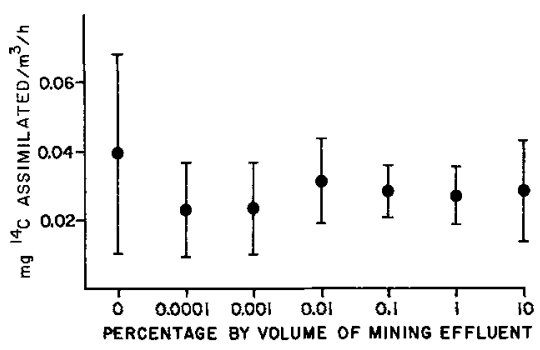

Fig. 9. Carbon-14 uptake in seawater at $50 \%$ light level inoculated with various concentrations of mining discharge effluent
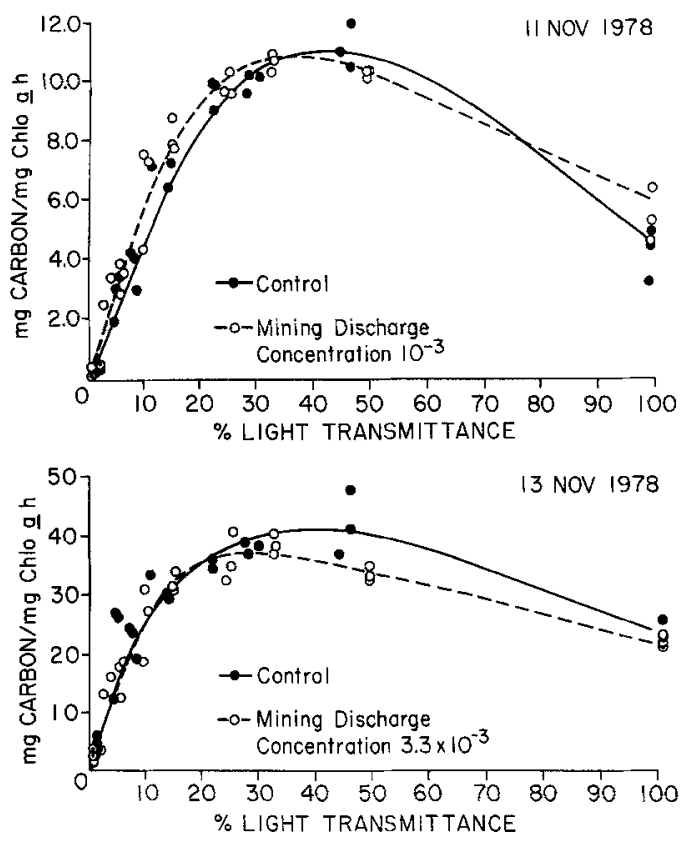

Fig. 10. Photosynthesis-irradiance curves for ambient surface water (control) and for ambient water inoculated with mining discharge at two different concentrations

(Fig. 9), and the effects of varying light conditions were examined for different concentrations of discharge (Fig. 10). Neither of these sets of experiments indicated significant differences in primary production that could be attributed to additional nutrient available from the discharge.

This series of experiments indicates that surface discharge will have some inhibiting effect on primary production, but this effect will be extremely localized and nonpersistent as the plume ages and is dispersed. There appears to be no evidence that the mining discharge can be considered a mini-upwelling, and no enhancement of primary production should be expected.

Another set of experiments was conducted to examine the effect of the surface plume on zooplankton. These experiments were prompted by two concerns: (1) the concern 
about mortality resulting from plume materials and (2) the general concern about possible introduction of metals into the food web.

Initial conclusions from field collection of zooplankton indicate no significant differences in mortality in the plume compared to ambient. Ongoing laboratory feeding experiments, in which zooplankters are fed varying concentrations of discharge, initially indicate no basic differences in comparison with control samples.

\section{SEA FLOOR IMPACT}

The sea floor effects of mining are all related to the action of the collector. The undisturbed sea floor is severely impacted by the collector. Bottom photographs and underwater video observation of the collector track indicate that the track is a zone of almost total destruction of benthic biota.

Associated with the collector contact zone is a region of relatively heavy resedimentation of much of the non-nodule material which is rejected by the collector near the sea floor. Analysis of a series of photographic traverses across the collector tracks indicates that this zone of heavy resedimentation is of the order of a hundred meters on each side of the collector track. Within this zone, resedimentation appears heavy enough to cause burial of some benthic biota and to cover the ambient sea floor to a degree which could reduce food availability for some organisms. Ultimate impact of these disturbances will be a function of the extent and duration of actual mining and, at present, can only be considered in a relative sense.

Although heaviest benthic impact is associated with the collector contact zone and the heavy resedimentation associated with it, some of the material resuspended by the collector is carried away by bottom currents in the form of a benthic plume. Detection of this benthic plume has been extremely difficult since its composition is poorly understood, and its movement depends upon near-bottom currents which are also not known. However, a combination of bottom-mounted arrays of nephelometers and current meters, deployed during some of the pilot-mining tests, and deep vertical casts to the sea floor resulted in several observations and samplings of the benthic plume. From the samples and measurements taken during the pilot-mining tests, the benthic plume is characterized by a thickness of the order of a few tens of meters and particulate concentrations of 15 to $150 \mu \mathrm{g} / \mathrm{l}$, compared with ambient values of approximately $10 \mu \mathrm{g} / \mathrm{l}$.

A major sampling of the benthic biota was initiated immediately prior to the pilotmining test at Site A. The sampling was centered on the planned mining sites and was initially designed to be repeated in order to assess pre-mining conditions, the immediate impact of the mining, and the conditions a year after the mining. The first two sets of samples were collected, but analysis through June of 1979 indicated no immediately obvious impact, and the plan for the one-year-after sampling has been abandoned. Analyses are continuing, and a final before/after comparison should be completed by the end of 1979.

In conclusion, the assessment of potential impact of mining has evolved from an initial pre-mining estimate. This original assessment has been modified on the basis of results from monitoring pilot-mining tests. It is expected that there will be additional tests before operational mining begins, and these will provide additional opportunities to examine the interactions between the mining systems and the marine environment. 


\section{LITERATURE CITED}

Bischoff, J. L. \& Piper, D. Z. (Eds), 1979, Marine geology and oceanography of the Pacific manganese nodule province. Plenum Press, New York, $842 \mathrm{pp}$.

Mustaffa, Z. \& Amann, 1978. Ocean mining and protection of the marine environment in the Red Sea. Offshore Technology Conference, Houston, Texas, May, 1978. - OTC Paper 3188.

Ozturgut, E., Anderson, G. C., Burns, R. E., Lavelle, J. W. \& Swift, S. A., 1978. Deep ocean mining of manganese nodules in the north Pacific: Pre-mining environmental conditions and anticipated mining effects. - NOAA Tech. Memorandum ERL MESA-33, 1-133. 